Oesophageal hold-up of tablets taken without water has been shown radiologically and is particularly likely if gastro-oesophageal reflux or hiatus hernia is present and the patient assumes a supine posture immediately after ingestion. ${ }^{3}$ Anatomically the oesophagus is indented by the aortic arch and the left main bronchus at about 24 and $28 \mathrm{~cm}$ respectively from the incisor teeth. In 18 cases reviewed by Collins et $a l^{1}$ in which the ulceration was probably due to direct contact between the tablet and mucosa and in which the site of the lesion was specified, the ulcer occurred in mid-oesophagus in 12 patients. It would seem reasonable to suggest that this site is vulnerable because of the anatomy of the area. With regard to tetracycline hydrochloride tablets in particular, the likelihood of mucosal damage is enhanced by the fact that these tablets dissolve in water to produce a highly acidic solution with $\mathrm{pH}$ as low as $2 \cdot 3 .^{2}$

We believe that the oesophageal ulceration in our patient was caused by a direct irritant effect from prolonged contact between oesophageal mucosa and a tetracycline hydrochloride tablet at about the level at which the left main bronchus crosses the oesophagus. It is advisable for patients using this antibiotic to take the evening dose some time before retiring and with adequate volumes of fluid.

We thank Professor J M Evanson for permission to report this case.

${ }^{1}$ Collins FJ, Matthews HR, Baker SE, Strakova JM. Drug-induced oesophageal injury. Br Med f 1979 ;i :1673-6.

2 Crowson TD, Head LH, Ferrante WA. Esophageal ulcers associated with tetracycline therapy. $\mathcal{F} A M A 1976 ; 235: 2747-8$.

${ }^{3}$ Evans KT, Roberts GM. Where do all the tablets go ? Lancet 1976;ii: 1237-9.

(Accepted 3 February 1981)

University Department of Medicine, Withington Hospital, Manchester M20 8LR

K S CHANNER, BSC, MRCP, senior house officer

D HOLLANDERS, MSC, MRCP, lecturer

\section{Severe hypoglycaemia during physical exercise and treatment with beta-blockers}

Despite important metabolic effects beta-blocking agents are usually well tolerated, but during metabolic stress important perturbations may occur. For instance, treatment with a non-selective beta-blocker leads to a slower rate of recovery from insulin-induced hypoglycaemia. ${ }^{1}$ Similarly, blood glucose concentrations are more rapidly reduced during prolonged physical exercise during treatment with a beta-blocker. ${ }^{2}$ Serious hypoglycaemia has occurred during prolonged exercise in patients receiving treatment with propranolol ${ }^{3}$ and pindolol. ${ }^{4}$

We report on a patient treated with alprenolol, a non-selective beta-blocker with intrinsic sympathomimetic activity, who developed serious hypoglycaemia during endurance exercise.

\section{Case report}

The patient was a previously healthy 61 -year-old man who was accustomed to jogging for one to two hours several times a week. Hypertension had been detected six years previously and the treatment finally adjusted to alprenolol $100 \mathrm{mg}$ twice daily and hydrallazine $25 \mathrm{mg}$ twice daily, with which his blood pressure was well regulated. He received no other medication and did not abuse alcohol. No diabetes was known in his family.

In 1979 he went cycling for about $1 \frac{1}{2}-2$ hours. He became increasingly fatigued and eventually lost consciousness. He recovered slowly on the way to a local hospital, where he was given glucose with immediate improvement.

Later in 1979 he went jogging after having had only a light lunch. After about 1-2 hours he felt increasing weakness and unsteadiness and then fell and could not rise. He was found unresponsive on the ground and brought to hospital. He had sinus bradycardia with a heart rate of around 30 beat $/ \mathrm{min}$. Blood pressure was $190 / 110 \mathrm{~mm} \mathrm{Hg}$. Blood glucose concentration was 1.9 $\mathrm{mmol} / \mathrm{l}(34 \mathrm{mg} / 100 \mathrm{ml})$. He was given intravenous glucose with immediate improvement: he became orientated and gave adequate answers though could not remember what had happened after he had fallen. Heart rate stabilised around 60 beats $/ \mathrm{min}$ and blood pressure around $160 / 90 \mathrm{~mm} \mathrm{Hg}$. Subsequent electrocardiography showed sinus rhythm with slightly raised ST intervals. Serum aspartate transaminase activity was slightly raised but then normalised; serum alanine transaminase activity remained normal. Lactate dehydrogenase isoenzyme electrophoresis showed increases in isoenzymes of myocardial origin. These enzyme changes combined with the discrete electrocardiographic changes suggest the possibility of a myocardial infarction, which might have developed during the hypoglycaemia. He had not felt any chest pain while jogging.

Fasting blood glucose concentrations while he was in hospital were all in the normal range (around $4.2 \mathrm{mmol} / 1(76 \mathrm{mg} / 100 \mathrm{ml})$ ) as was the fasting insulin concentration $(3.0 \mathrm{mU} / \mathrm{l})$.

He was subsequently discharged from hospital taking metoprolol (a cardioselective beta ${ }_{1}$-blocking agent) $100 \mathrm{mg}$ twice daily and hydrallazine 25 mg twice daily. He was told to eat regularly, particularly when taking exercise. He remained well and resumed his previous level of physical activity.

\section{Comment}

In this patient one suspected and one verified episode of severe, symptomatic hypoglycaemia occurred in association with prolonged exercise. There was no evidence of an insulinoma or any other known cause of hypoglycaemia apart from the endurance exercise.

During prolonged, submaximal exercise glucose and free fatty acids are mainly taken up from the blood stream and used by the working muscles. ${ }^{5}$ Splanchnic glucose production cannot keep pace with the increased rate of use, leading to a fall in blood glucose concentrations. ${ }^{5}$ With beta-blockade, particularly with non-selective agents, this fall occurs more rapidly, ${ }^{2}$ probably because of impaired glucose production in the liver and a greater dependency on glucose as substrate since the release of free fatty acids from the adipose tissue is impaired by these agents. ${ }^{1}$

The haemodynamic pattern in our patient (bradycardia and raised blood pressure) was similar to that reported previously in patients with hypoglycaemia receiving concomitant treatment with a nonselective beta-blocker. ${ }^{1}$ Thus a potentially serious reaction may occur during prolonged physical exercise and concomitant beta-blockade. Patients treated with beta-blockers should be particularly careful to ingest suitable energy-giving food when participating in endurance exercise.

1 Lager I, Blohmé G, Smith U. Effect of cardioselective and non-selective beta-blockade on the hypoglycaemic response in insulin-dependent diabetics. Lancet 1979 ;i:458-62.

2 Galbo H, Holst JJ, Christensen NJ, Hilsted J. Glucagon and plasma catecholamines during beta-receptor blockade in exercising man. f Appl Physiol 1976;40:855-63.

3 Aksnes EG. Beta-blokkare-farlig for skiløpere ? Tidsskrift for den Norske Laegeforening 1977;97:576.

4 Uusitupa M, Aro A, Pietikäinen M. Severe hypoglycaemia caused by physical strain and pindolol therapy. Ann Clin Res 1980;12:25-7.

5 Wahren J, Felig P, Hagenfeldt L. Physical exercise and fuel homeostasis in diabetes mellitus. Diabetologia 1978;14:213-22.

(Accepted 9 February 1981)

Departments of Medicine I and II, Sahlgren's Hospital, University of Göteborg, Göteborg, Sweden

G HOLM, MD, associate professor of internal medicine J HERLITZ, MD, resident

U SMITH, MD, associate professor of internal medicine

\section{Educational value of printed information for patients with hypertension}

Printed information booklets are widely used to inform patients about their condition. We investigated the educational value of one such booklet among patients with high blood pressure.

\section{Patients, methods, and results}

One hundred consecutive patients attending a blood pressure clinic were randomised into two groups, one of which received a booklet about hypertension $^{1}$ and the other attended the clinic as before. The booklet explained the reasons for controlling high blood pressure, the need for continuous treatment even in the absence of symptoms, the importance of cardiovascular 
risk factors, and details of the main groups of antihypertensive drugs. An energy chart was included, as were tables detailing the cholesterol and potassium contents of selected foods and ideal body weights. Finally, there was a section for recording blood pressure values and drug prescriptions. Patients were told only that this was a booklet about high blood pressure and were asked to bring it with them when they visited the clinic or their family doctor so that their blood pressure and drug treatment could be recorded. The clinic doctors were unaware of the group to which each patient had been allocated as the booklet was completed by a nurse during the three months of the study.

After three months all 100 patients were asked to complete a questionnaire containing 40 questions. These included six direct questions about the patients' blood pressure, weight, and drug treatment. The remaining 34 questions allowed the patients to answer "true," "false," or "don't know" to statements about the nature and treatment of hypertension and the dietary information contained in the booklet. The questionnaire was completed immediately before the patient left the hospital to prevent patients discussing it in the waiting area.

Ninety-three patients completed the study, of whom 46 had received the booklet. There was no significant difference between the groups in the duration of attendance at the clinic or age (mean age 51.2 years). The mean number of correct answers given by patients using the booklet was $23.9 \pm 0.8$ and $18.1 \pm 0.8$ by the controls $(p<0.001)$, the maximum score possible being 40 .

The table shows results for individual questions. More patients from the booklet group could name their drugs and last recorded blood pressure.

Number of correct answers to questions concerning patients' own state and general questions about hypertension

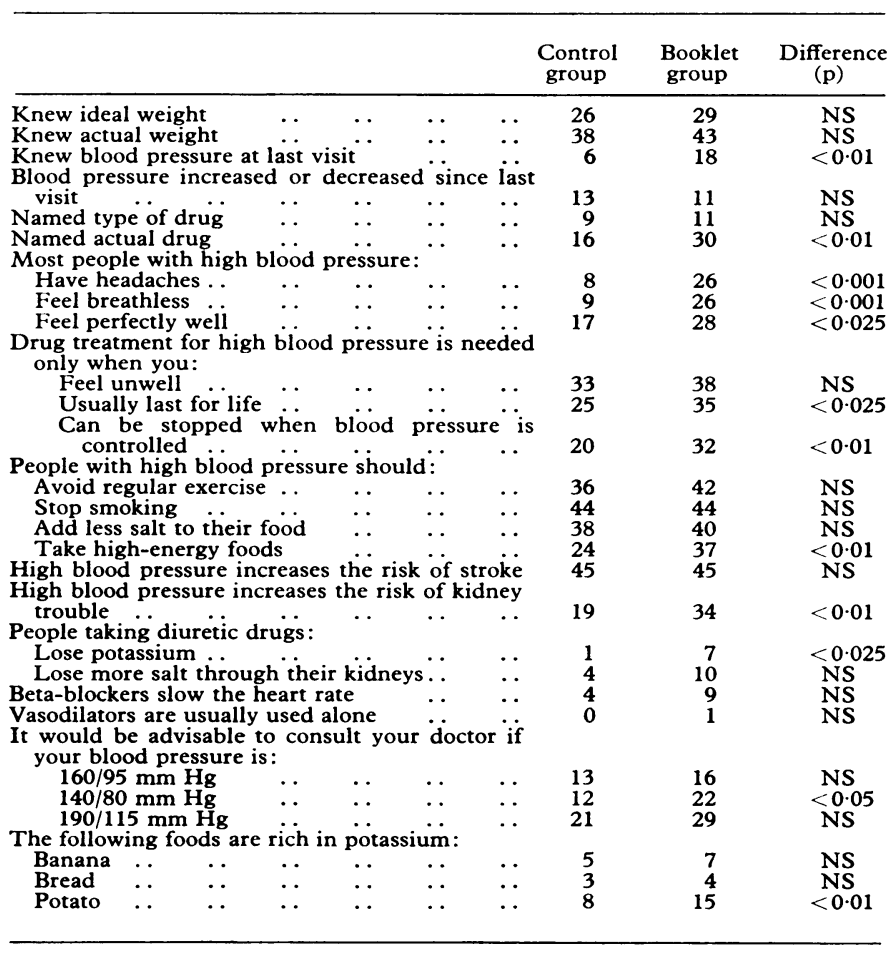

They were more often aware of the asymptomatic nature of the condition, the need for life-long treatment, the association between hypertension and kidney trouble, and the cholesterol and energy values of selected foods. There was no significant difference, however, between the groups in knowledge of their weight, whether their blood pressure had increased or decreased since their last visit, and the pharmacological classification of their drugs. Both groups were equally aware of the relations of exercise, smoking, salt, and stroke to hypertension, but few understood the importance of various values of blood pressure and virtually none understood the side effects of drugs.

\section{Comment}

Since this study was single-blind and no explanation of the text was given to the patients the increased knowledge of the study group about some important aspects of hypertension may be attributed directly to the booklet. Some of the information provided, however, particularly that relating to drugs, was not understood. This emphasises the need to keep the text of such booklets simple, in keeping with recent recommendations, ${ }^{2}$ and patients should be encouraged to discuss their reading with their doctor.

The study was not designed to test for differences in compliance or the outcome of treatment, but analysis shows that there was no appreciable difference in blood pressure or weight between the groups. While educating patients does not in itself improve compliance, patients should have at least some basic understanding of their treatment. ${ }^{3}$ Finally, the booklet also serves as an effective means of communication between the clinic and family doctor and this must ultimately contribute to better patient care. ${ }^{4}$

We are pleased to acknowledge the support of the Irish Heart Foundation and the Department of Health.

1 O'Hanrahan M, O'Malley K, O'Brien ET. Printed information for the lay public on cardiovascular disease. $\mathrm{Br}$ Med $71980 ; 281: 597-9$.

2 Anonymous. Drug information for patients: keep it simple. $\mathrm{Br} \mathrm{Med} \mathfrak{F}$ $1980 ; 280: 1393$.

${ }^{3}$ Bullen MU. What patients with hypertension should know about their medication. Drugs 1980;19:373-9.

${ }^{4}$ Ezedum S, Kerr DNS. Collaborative care of hypertension using a shared record. $\mathrm{Br} \mathrm{Med} \mathcal{F}$ 1977;ii:1402-3.

(Accepted 11 February 1981)

Blood Pressure Clinic, The Charitable Infirmary, Dublin 1, and Department of Clinical Pharmacology, Royal College of Surgeons, St Stephen's Green, Dublin 2

M LAHER, LRCPI, LRCSI, research fellow

K O'MALLEY, MD, FRCPI, professor of clinical pharmacology

E O'BRIEN, MRCP, FRCPI, consultant physician

M O'HANRAHAN, BSC, MPSI, research pharmacist

C O'BOYLE, MRCPI, DCH, research fellow

\section{Biphasic sulphinpyrazone-warfarin interaction}

Previous reports have provided evidence that sulphinpyrazone can potentiate the anticoagulant effect of warfarin. ${ }^{12}$ We recently observed a woman in whom the prolonged addition of sulphinpyrazone caused a biphasic interaction with warfarin-that is, potentiation followed by antagonism.

\section{Case report}

A 37-year-old woman with recurrent venous thrombosis and pulmonary embolism was treated with about $15 \mathrm{mg}$ warfarin daily for one month. Notwithstanding this her prothrombin time (Thrombotest, Nyegaard and $\mathrm{Co}$, Oslo) was near to but not within the therapeutic range. Owing to the recurrence of venous thrombosis we added to this regimen $200 \mathrm{mg}$ sulphinpyrazone three times a day $(10 \mathrm{mg} / \mathrm{kg}$ body weight/day). This drug was chosen for its reported efficacy in controlling the recurrence of venous thrombosis. ${ }^{3}$ As expected, the dose of warfarin required fell rapidly after sulphinpyrazone was started and then remained between 7.5 and $10 \mathrm{mg} /$ day for about 15 days (figure). Thereafter the warfarin requirement began to rise progressively until $30 \mathrm{mg} /$ day was not enough to keep the result of a Thrombotest within the therapeutic range. An increase in sulphinpyrazone dosage (200 $\mathrm{mg}$ four times daily) resulted in only a transient reduction in Thrombotest values. At this point sulphinpyrazone was stopped: the warfarin requirement fell rapidly to $5 \mathrm{mg} /$ day and after a few days had returned to the values seen before the addition of sulphinpyrazone. No drugs other than sulphinpyrazone or warfarin were administered during the observation period. There was no reason to doubt the patient's compliance with either medication. Appreciable changes in diet were carefully avoided.

\section{Comment}

Displacement of a drug from its binding protein allows the drug to act but also facilitates its metabolism or excretion. ${ }^{4}$ Both phenylbutazone $^{4}$ and sulphinpyrazone, ${ }^{5}$ which are chemically related, can displace coumarins from their albumin binding sites and initially induce a fall in the dosage of warfarin required. When phenylbutazone is administered long term it induces enhanced hepatic metabolism of warfarin and thus a biphasic effect is seen during its administration." Even though plasma concentrations of warfarin were not measured, we believe that in our case this same biphasic interaction occurred 